\title{
Design of High Frequency Current-Mode Continuous-Time Filter using CMOS Current-Mirrors
}

\author{
Agha A. Husain ${ }^{1}$, Manveen S. Chadha ${ }^{2}$, Ashish Gupta ${ }^{3}$, Amendra Bhandari ${ }^{4}$ \\ 1, 2,3,4 (Department of Electronics and Communication Engineering, I.T.S Engineering College, Plot No: 46, \\ Knowledge Park-III, Greater Noida, (U.P.), INDIA.)
}

\begin{abstract}
Interest in current-mode (CM) filters has been growing due to the fact that current-mode devices have wider dynamic range, improved linearity, and extended bandwidth compared with voltage-mode devices. The commonly used circuit techniques for designing current-mode filters are mainly classified into two categories. One technique is based on the transformation of the voltage-mode circuits to current-mode ones, such as the adjoint network, the RC:CR dual transformation and the inverse-complementary network, etc. The other technique uses the direct current-mode integrators as the basic cell of the design biquad and higher-order filters. Depending on the technology chosen, the frequency range of transconductance circuits extends to $>50$ $\mathrm{MHz}(\mathrm{CMOS}),>500 \mathrm{MHz}$ (bipolar) or even to $>1 \mathrm{GHz}$ (GaAs) so that the design of high-frequency continuoustime telecommunication circuits becomes feasible. In addition, the wider useful bandwidth of transconductances coupled with the reduced effects of circuit and device parasitics on filter performance result in far higher operating frequencies at which the circuits can function. The proposed circuit presented in the paper was tested

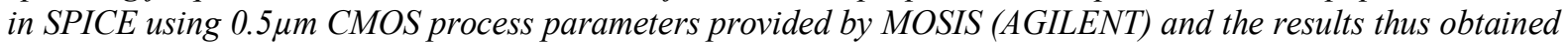
were in accordance with the theoretical values.
\end{abstract}

Keywords: Current-mode filters, Current-Adders, Current-Integrators, CMOS Current-Mirrors, Second Order Filter.

\section{Introduction}

A filter ([9], [10], [13]) is a two-port network that shapes the spectrum of the input signal in order to obtain an output signal with the desired frequency content. Thus, a filter has pass bands where the frequency components are transmitted to the output and stop bands where they are rejected. Traditionally, such circuits working in the continuous-time domain have been designed as resistively terminated lossless LC filters where resonance could be employed to achieve complex poles and the desired steep transition regions between pass bands and stop bands. With the growing pressure towards microminiaturization, inductors were found to be too bulky so that designers started to replace passive RLC filters by active RC circuits where gain, obtained from operational amplifiers, together with resistors and capacitors in feedback networks, was used to achieve complex poles. Active filters based on the ubiquitous op-amp have found wide acceptance throughout the industry over the past and their mature development and technology guarantee their continued popularity in many applications.

One disadvantage of op-amp based active RC filters is the limited frequency range over which these circuits can be used: the finite bandwidth of op-amps usually constrains the application to be below $100 \mathrm{kHz}$ with performance becoming deteriorating with increasing frequency. For operation in $\mathrm{MHz}$ range the main design challenges for analog filters are: (i) Reliable high-frequency performance. (ii) Automatic on-chip tuning against fabrication tolerances and changing operating conditions. Therefore, for high frequency applications the output was obtained in the form of current where the output current was given by:

$\mathrm{I}_{\text {out }}=\mathrm{g}_{\mathrm{m}} \mathrm{V}_{\text {in }}$

where, $\mathrm{g}_{\mathrm{m}}$ is the transconductance parameter provided by the active device.

For application in continuous-time filters ([1], [2], [5]) transconductance should satisfy the following main properties: (i) Circuits must be simple, linear and have a wide frequency response. (ii) Must have large input and output impedances to prevent undesirable interactions and simplify circuit design. (iii) They should preferably work with low-voltage power supplies to conserve power and to be compatible with the prevalent digital technologies on the same chip. (iv) Their transconductance parameter must depend on some dc bias voltage or current to facilitate electronic tuning against environmental or processing variations.

In many useful active simulations of filters, we may even insist that all the transconductors are identical and all capacitors grounded for specially simple IC layout and processing, and implementation of integrated analog filters based on analog gate arrays appears a distinct possibility. In addition, the wider useful bandwidth of transconductances coupled with the reduced effects of circuit and device parasitics on filter performance result in far higher operating frequencies at which the circuits can function. 


\section{Proposed Circuits}

Current-mode signal processing ([3], [5], [9]) is quite attractive for low power supply voltage operation and high frequency application. In this paper design of current-mode filters using nMOS transistor current mirrors and pMOS transistor current sources as an active load, as reported in [2], which is a counterpart of the MOS realization [4], are presented. In that design, pMOS transistors were only used for DC current sources which provide bias currents to each current mirror and also behave as active loads of the current mirror.

The currents of these sources must match each other and also match with DC currents of each current mirror to give a proper DC bias to transistors. However the matching is sometimes difficult due to the parameter mismatch between nMOS and pMOS transistors. In this paper, design of CMOS continuous-time filters using nMOS and pMOS transistor current mirrors is presented. In monolithic integrated circuits, it has long been prohibited to use pMOS transistor for signal processing except for DC biasing purpose because of the poor characteristics of pMOS transistors, especially because of their poor frequency performances. However, due to development of semiconductor technology, pMOS transistors with performances comparable to nMOS transistors are now available and can be used as signal processing elements. Using pMOS transistors as signal processing elements, circuits can be simplified and problems resulting from difficulty of DC biasing can be avoided.

Using integrators and adders, any kind of transfer function can be realized; therefore the method can easily be applied for wide range of filter realization. Generally, monolithic integrated filters have a difficulty on setting the filter frequency due to absolute value error of elements. In these filters, the tuning of the filter frequency can easily be achieved by adjusting the current of a single DC current source.

\subsection{Realization of Current-Mode Second-Order Low Pass Active Filter}

Among the numerous techniques available for active RC filter realizations, we present cascade method of second order filter realization which is quite popular and can generate any type of transfer function. As the first-order low pass section can be realized by the lossy integrator of Fig. 2 and Fig. 4 [8], the second order section is presented here. Figure-1 shows the block diagram of the current-mode second-order low Pass section using a lossless and a lossy integrator of Fig. 6 and Fig. 4 respectively [8].

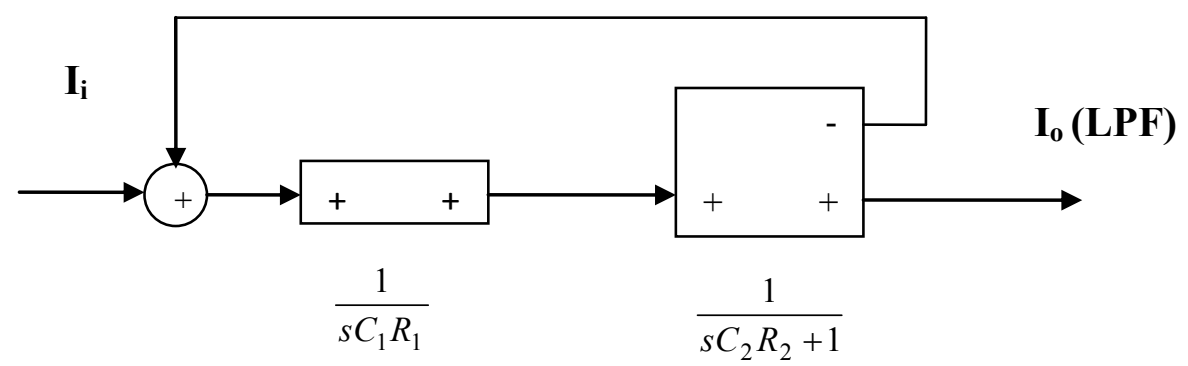

Fig. 1: Block Diagram Of Second-Order Section

The current transfer function of Figure-1 is given by

$$
T_{2}(s)=\frac{1}{s^{2} C_{1} C_{2} R_{1} R_{2}+s C_{1} R_{1}+1}
$$

On comparing equation-2.1 with the standard form of second-order low Pass transfer function given by equation-2.2 we obtain the following results for the cut-off frequency $\left(\omega_{0}\right)$ and the quality factor $(\mathrm{Q})$ given by equation-2.3.

$$
T_{L P}(s)=\frac{\omega_{o}^{2}}{s^{2}+\frac{\omega_{o}}{Q} s+\omega_{o}^{2}}
$$

where,

$$
\omega_{o}=\sqrt{\frac{1}{C_{1} C_{2} R_{1} R_{2}}} \quad \text { and } \quad Q=\sqrt{\frac{C_{2} R_{2}}{C_{1} R_{2}}}
$$


Figure-2 shows the practical realization of the filter where the lossless integrator of Type-I shown in Fig. 5 [8] and the complementary type of the simplified lossy integrator shown in Fig. 4 [8] are used and the DC bias current source $\mathrm{I}_{\mathrm{B}}$ of simplified lossy integrator of Fig. 4 [8] is eliminated by the use of the output DC bias current of $\mathrm{M}_{4}$ of Figure- 2 .

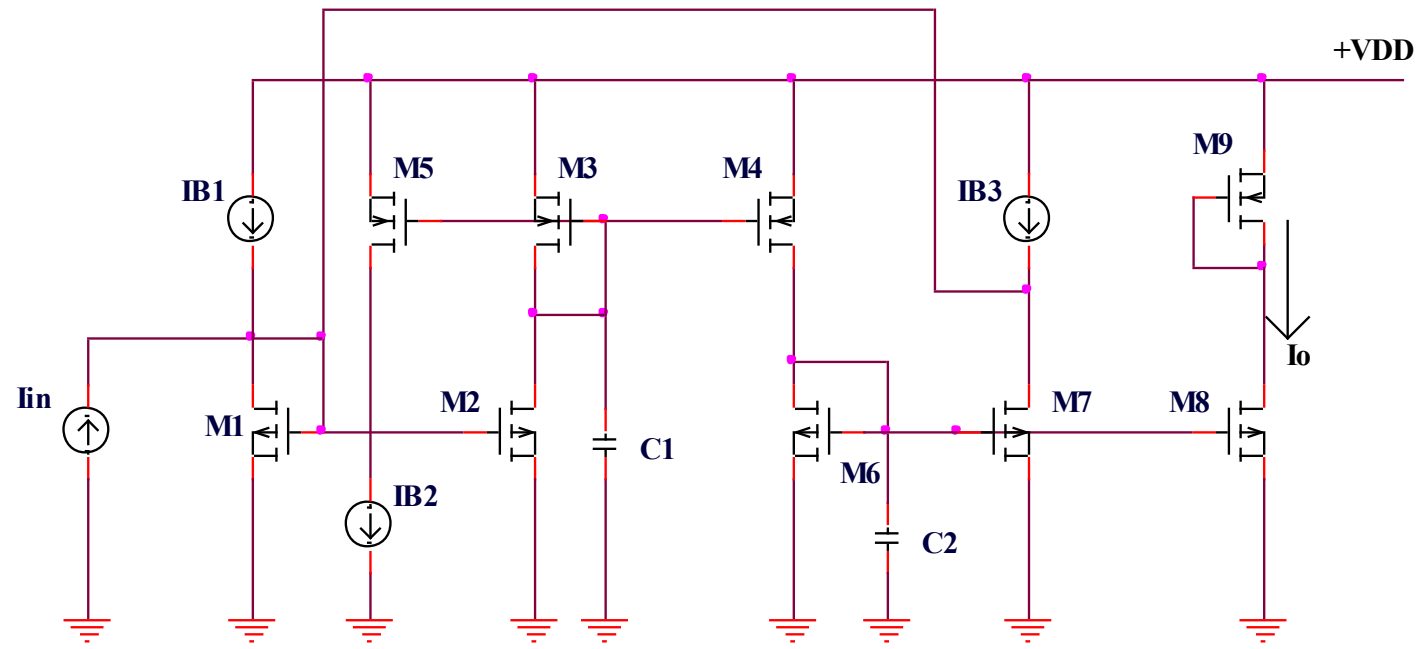

Fig. 2: Current Mode Second Order Section

Assuming that all transistors of Figure-2 have an equal aspect ratio's that is, all current mirrors have a unity gain, we have the following transfer function:

$$
T_{2}(s)=\frac{1}{s^{2} C_{1} C_{2} R^{2}+s C_{1} R+1}
$$

Since the DC bias currents of all transistors are equal to the bias current $\mathrm{I}_{\mathrm{B}}$, the resistances are given by,

$$
R_{1}=R_{2}=R \cong \frac{k T}{q} \frac{1}{I_{B}} \cong \frac{0.0259}{I_{B}}
$$

and also we have, $\mathrm{I}_{\mathrm{B} 3}=\mathrm{I}_{\mathrm{B} 2}=\mathrm{I}_{\mathrm{B} 1}=\mathrm{I}_{\mathrm{B}}$.

Equation-2.5 shows that $I_{B}$ controls both $R_{1}$ and $R_{2}$ simultaneously and equation- 2.3 shows that the simultaneous control of $R_{1}$ and $R_{2}$ does not affect the $Q$ factor. Thus we can control the cut-off frequency of the filter $\omega_{\mathrm{o}}$ without changing the $\mathrm{Q}$ factor.

While cascading the first-order or second-order sections, the DC bias current $\mathrm{I}_{\mathrm{B}}$ of each stage can be provided from the output of the former stage and therefore a single DC bias current source is only required at the front stage of filters.

\section{Simulation Results}

The workability of the proposed circuits were tested and verified in SPICE using $0.5 \mu \mathrm{m}$ CMOS process parameters provided by MOSIS (AGILENT) as listed in Table-1.

\begin{tabular}{|c|c|}
\hline TRANSISTOR & PROCESS PARAMETERS \\
\hline nMOS & 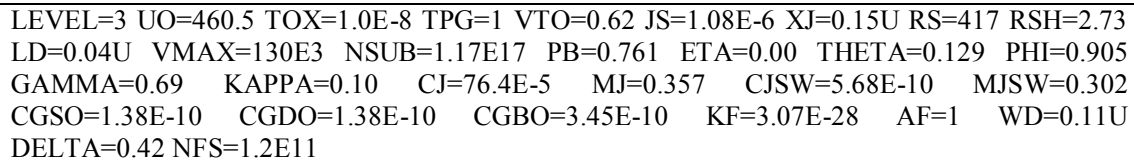 \\
\hline pMOS & $\begin{array}{l}\mathrm{LEVEL}=3 \mathrm{UO}=100 \mathrm{TOX}=1.0 \mathrm{E}-8 \mathrm{TPG}=1 \mathrm{VTO}=0.58 \mathrm{JS}=0.38 \mathrm{E}-6 \quad \mathrm{XJ}=0.10 \mathrm{U} \text { RS }=886 \mathrm{RSH}=1.81 \\
\mathrm{LD}=0.03 \mathrm{U} \text { VMAX }=113 \mathrm{E} 3 \quad \mathrm{NSUB}=2.08 \mathrm{E} 17 \quad \mathrm{~PB}=0.911 \quad \mathrm{ETA}=0.00 \quad \mathrm{THETA}=0.120 \quad \mathrm{PHI}=0.905 \\
\mathrm{GAMMA}=0.76 \mathrm{KAPPA}=2 \mathrm{CJ}=85 \mathrm{E}-5 \mathrm{MJ}=0.429 \mathrm{CJSW}=4.67 \mathrm{E}-10 \mathrm{MJSW}=0.631 \mathrm{CGSO}=1.38 \mathrm{E}-10 \\
\mathrm{CGDO}=1.38 \mathrm{E}-10 \mathrm{CGBO}=3.45 \mathrm{E}-10 \mathrm{KF}=1.08 \mathrm{E}-29 \mathrm{AF}=1 \mathrm{WD}=0.14 \mathrm{U} \text { DELTA }=0.81 \mathrm{NFS}=0.52 \mathrm{E} 11\end{array}$ \\
\hline
\end{tabular}

\section{TABLE-1: CMOS PROCESS PARAMETERS}

For the circuit shown in Figure 2 the ac analysis were carried out with the value of dc bias current $\mathrm{I}_{\mathrm{B} 1}=$ $\mathrm{I}_{\mathrm{B} 2}=\mathrm{I}_{\mathrm{B} 3}=45 \mu \mathrm{A}, \mathrm{C}_{1}=\mathrm{C}_{2}=1 \mathrm{pF},(\mathrm{W} / \mathrm{L})_{\mathrm{P}}$ ratio $=5 \mu \mathrm{m} / 1 \mu \mathrm{m},(\mathrm{W} / \mathrm{L})_{\mathrm{N}}$ ratio $=1 \mu \mathrm{m} / 1 \mu \mathrm{m}$ and supply voltage $\mathrm{V}_{\mathrm{DD}}=$ $2.5 \mathrm{~V}$. The value of cut-off frequency is found to be $\mathrm{f}_{\mathrm{O}}=19.259 \mathrm{MHz}$ which is very well in agreement with the calculated theoretical value of $\mathrm{f}_{\mathrm{O}}=19.3 \mathrm{MHz}$. SPICE simulation results are shown in Figure-3. 


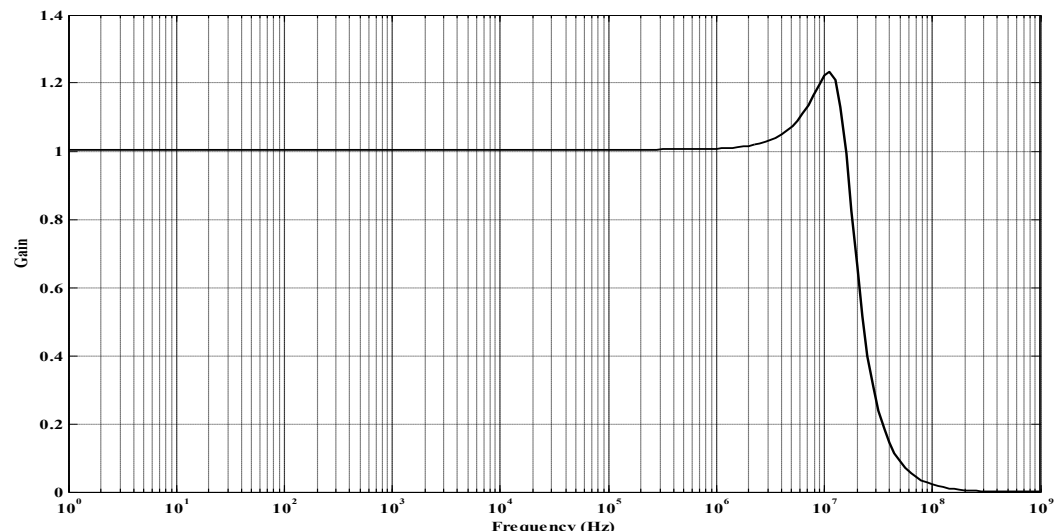

Fig. 3: Low Pass Response of Second Order Current-mode Continuous-time Filter

Figure-4 shows the change in the value of cut-off frequency with change in the value of capacitor $\mathrm{C}_{1}$ where the value of capacitor was varied from $1 \mathrm{pF}$ to $20 \mathrm{pF}$.

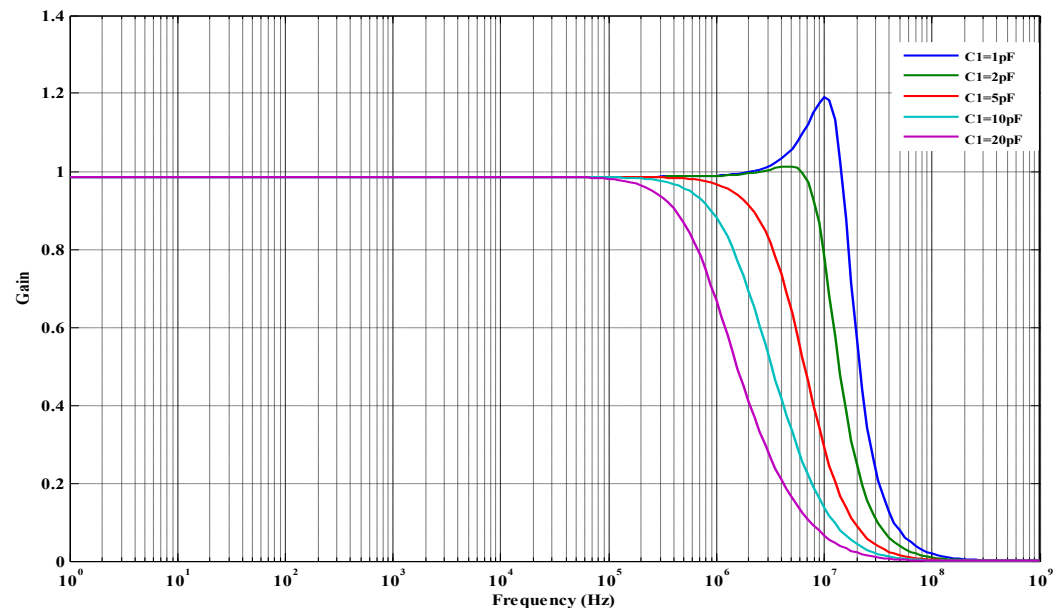

Fig. 4: Variation in the cut-off frequency with change in capacitor value $C_{1}$

Figure- 5 shows the change in the value of cut-off frequency as well as the quality factor with change in the value of capacitor $\mathrm{C}_{2}$ where the value of capacitor was varied from $1 \mathrm{pF}$ to $20 \mathrm{pF}$.

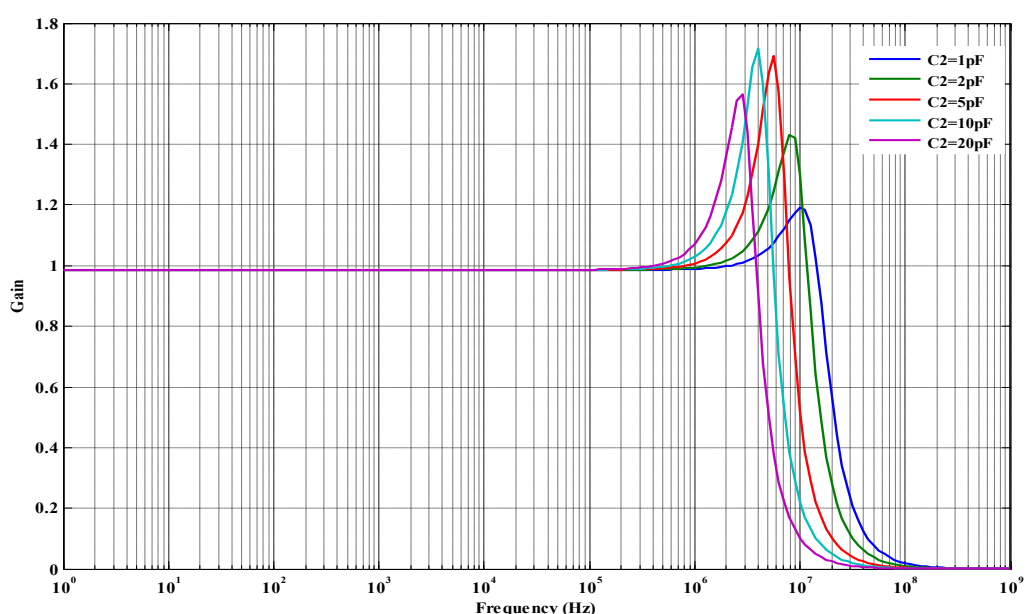

Fig. 5: Variation in the cut-off frequency and Quality Factor with change in capacitor value $C_{2}$ 
Figure- 6 shows the change in the value of gain with change in the value of bias current when varied from $10 \mu \mathrm{A}$ to $45 \mathrm{uA}$.

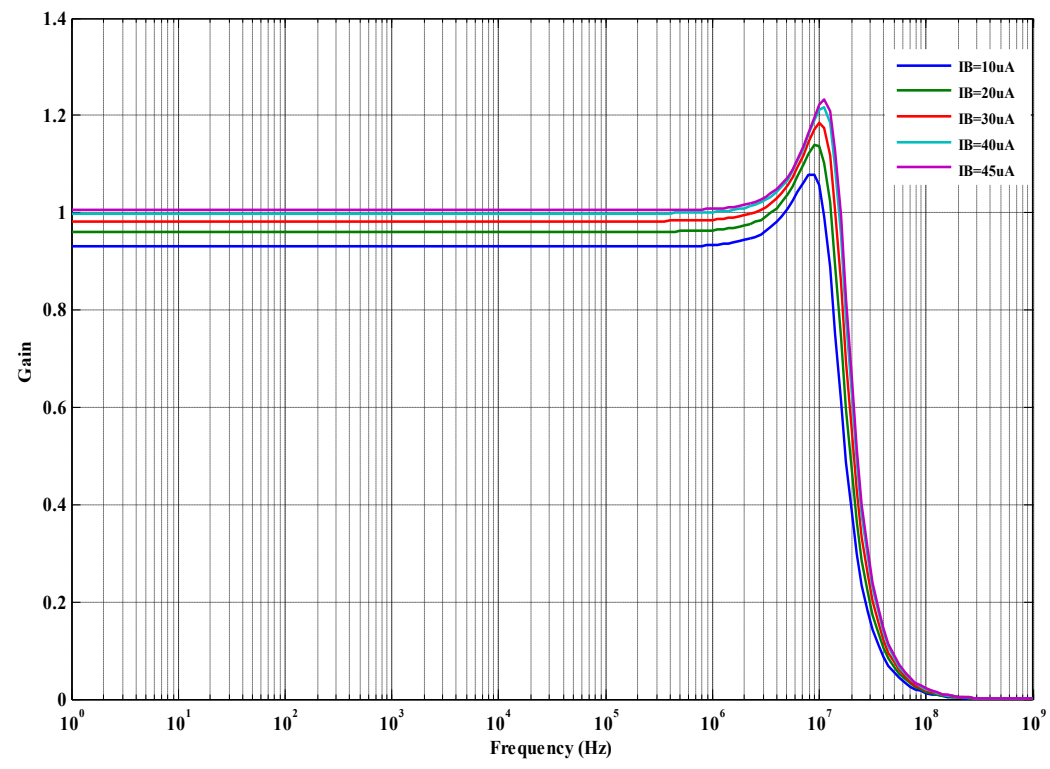

Fig. 6: Variation in the Gain with change in bias current $I_{B}$

\section{Conclusion}

In the given paper high frequency second order current-mode filter have been presented which can be used to form higher order active filters. This active filter is quite suitable for the realization in high frequencies of more than $10 \mathrm{MHz}$ and this filter can operate at a voltage as low as $1.5 \mathrm{~V}$ or less. It has been also verified that variations in the value of either the capacitor or the bias current improves the gain as well as the operating frequency of the filters. The frequency of this filter can be easily and widely controlled by a single DC bias current and thus provides good tunability. All the circuits were tested using SPICE and the verified results confirms the theoretical values.

\section{References}

[1] R. J. Angulo, and E. S. Sinencio, Active compensation of operational transconductance amplifier using partial positive feedback, IEEE J. of Solid-state Circuits, 25, 1990, 1024-1028

[2] J. C. Ahn, and N. Fujii, Current-mode continuous-time filters using complementary current mirror pairs, IEICE Trans Fundamentals, E79-A(2), 1996, 168-175.

[3] R. J. Angulo, M. Robinson, and E. S. Sinencio, Current-mode continuous-time filters: two design approaches, IEEE Tran. On Circuits and Systems, 39(5), 1992, 337-341.

[4] S. S. Lee, R. H. Zele, D. J. Allstot, and G. Liang, A continuous-time current-mode integrator, IEEE Trans. On Circuits and Systems, 39, 1991, 1236-1238.

[5] S. S. Lee, R. H. Zele, D. J. Allstot, and G. Liang, CMOS continuous-time current-mode filters for high frequency applications, IEEE J. Solid State Circuits, 28(3), 1993, pp. 323-329

[6] C. Toumazou, and F. J. Lidgey, Novel bipolar differential input/output current-controlled current source, Electronic Letters, 21, 1985, 199-200.

[7] B. Wilson, Analogue current-mode circuits, International Journal of Electronics Engineering Education, 26, 1989, 206-233

[8] Amendra Bhandari, Agha A. Husain, Manveen S. Chadha, Ashish Gupta, Lossy and Lossless Current-mode Integrators using CMOS Current Mirrors, International Journal of Engineering Research and Development, 9(3), 2013, 34-41.

[9] C. Toumazou, F. J. Lidgey, and D. G. Haigh, Analogue IC Design: The current-mode approach', Peter Peregrinus Ltd., 1990.

[10] R. Schaumann, M. S. Ghausi, and K. R. Laker, Design of Analog Filters: Passive, Active RC, and Switched Capacitor', Englewood Cliffs, NJ, Prentice Hall, 1989

[11] C. Toumazou, F. J. Lidgey, and P. Y. K. Cheung, Current-mode analogue signal processing circuits-a review of recent developments, IEEE International Symposium on Circuits and Systems, Portland, USA, vol.3, 1989, 1572-1575.

[12] M. Desai, P. Aronhime, and K. Zurada, Current-mode network transformation, IEEE Proc. ISCAS 1994, $599-602$.

[13] D. G. Haigh, and J. T. Taylor, Continuous-time and switched capacitor monolithic filters based on current and charge simulation, IEEE International Symposium on Circuits and Systems, Portland, USA, 3, 1989, 1580-1583.

[14] G. H. Wang, K. Watanabe, and R. Fukui, An extended dual transformation approach to current-mode circuit synthesis, IEEE Proc. ISCAS, 1990, 2294-2295. 